\title{
Some remarks on regularized nonconvex variational inequalities
}

\author{
Javad Balooee ${ }^{1}$ and Jong Kyu Kim² ${ }^{2 *}$
}

\author{
"Correspondence: \\ jongkyuk@kyungnam.ac.kr \\ 2Department of Mathematics \\ Education, Kyungnam University, \\ Changwon, Gyeongnam 631-701 \\ Korea \\ Full list of author information is \\ available at the end of the article
}

\begin{abstract}
In this paper, we investigate and analyze the nonconvex variational inequalities introduced by Noor in (Optim. Lett. 3:411-418, 2009) and (Comput. Math. Model. $21: 97-108,2010)$ and prove that the algorithms and results in the above mentioned papers are not valid. To overcome the problems in the above cited papers, we introduce and consider a new class of variational inequalities, named regularized nonconvex variational inequalities, instead of the class of nonconvex variational inequalities introduced in the above mentioned papers. We also consider a class of nonconvex Wiener-Hopf equations and establish the equivalence between the regularized nonconvex variational inequalities and the fixed point problems as well as the nonconvex Wiener-Hopf equations. By using the obtained equivalence formulations, we prove the existence of a unique solution for the regularized nonconvex variational inequalities and propose some projection iterative schemes for solving the regularized nonconvex variational inequalities. We also study the convergence analysis of the suggested iterative schemes under some certain conditions.
\end{abstract}

MSC: 47H05; 47J20; 49J40; 90C33

Keywords: regularized nonconvex variational inequalities; nonconvex sets; iterative algorithm; prox-regularity; convergence analysis

\section{Introduction}

Variational inequality theory, introduced by Stampacchia [1], has become a rich source of inspiration and motivation for the study of a large number of problems arising in economics, finance, transportation, network and structural analysis, elasticity and optimization. Many research papers have been written lately, both on the theory and applications of this field. Important connections with main areas of pure and applied sciences have been made; see, for example, [2-4] and the references cited therein. The development of variational inequality theory can be viewed as the simultaneous pursuit of two different lines of research. On the one hand, it reveals the fundamental facts on the qualitative aspects of the solution to important classes of problems; on the other hand, it also enables us to develop highly efficient and powerful new numerical methods to solve, for example, obstacle, unilateral, free, moving and complex equilibrium problems. One of the most interesting and important problems in variational inequality theory is the development of an efficient numerical method. There is a substantial number of numerical methods including projection method and its variant forms, Wiener-Hopf (normal) equations, auxiliary principle, and descent framework for solving variational inequalities and complementar-

\section{空 Springer}

(c)2013 Balooee and Kim; licensee Springer. This is an Open Access article distributed under the terms of the Creative Commons Attribution License (http://creativecommons.org/licenses/by/2.0), which permits unrestricted use, distribution, and reproduction in any medium, provided the original work is properly cited. 
ity problems. For the applications, physical formulations, numerical methods and other aspects of variational inequalities, see [1-22] and the references therein.

The projection method and its variant forms represent an important tool for finding an approximate solution of various types of variational and quasi-variational inequalities, the origin of which can be traced back to Lions and Stampacchia [19]. The projectiontype methods were developed in 1970s and 1980s. The main idea in this technique is to establish the equivalence between the variational inequalities and the fixed point problems using the concept of projection. This alternative formulation enables us to suggest some iterative methods for computing an approximate solution.

It is worth mentioning that most of the results regarding the existence and iterative approximation of solutions to variational inequality problems have been investigated and considered so far to the case where the underlying set is a convex set. Recently, the concept of convex set has been generalized in many directions, which has potential and important applications in various fields. It is well known that the uniformly prox-regular sets are nonconvex and include the convex sets as special cases. This class of uniformly prox-regular sets has played an important part in many nonconvex applications such as optimization, dynamic systems and differential inclusions. For more details, see, for example, $[9,10,13$, $14,21,23,24]$.

Very recently, Noor $[25,26]$ has introduced and considered a new class of variational inequalities, the so-called nonconvex variational inequalities (NVI) on the uniformly proxregular sets. He has also introduced a class of Wiener-Hopf equations in [26]. The author has asserted that NVI $(2.1)$ from $[25,26]$ is equivalent to the fixed point problem (3.1) from $[25,26]$ as well as the Wiener-Hopf equation (2.5) from [26]. Then, he used the fixed point formulation (3.1) from [25, 26] and the equivalence formulations (4.1) and (4.2) from [26], and suggested some iterative schemes for solving NVI $(2.1)$ from $[25,26]$. He also studied the convergence analysis of the suggested iterative methods under certain conditions.

In this paper, we establish that the equivalence formulation (3.1), used by Noor in [25, $26]$, is not correct. That is, Lemma 3.1 in [25, 26], which is the main tool to suggest the algorithms and to prove the strong convergence of the sequences generated by the proposed iterative algorithms in $[25,26]$, is incorrect. Consequently, the algorithms and results in $[25,26]$ are not valid. To overcome these problems in $[25,26]$, we introduce and consider a new class of variational inequalities, termed the regularized nonconvex variational inequalities (RNVI), instead of the class of NVI $(2.1)$ from $[25,26]$. We also consider a class of nonconvex Wiener-Hopf equations (NWHE) and establish the equivalence between RNVI and the fixed point problems as well as NWHE. By using the obtained equivalence formulations, we prove the existence of a unique solution for RNVI and propose some projection iterative schemes for solving RNVI. We also study the convergence analysis of the suggested iterative schemes under some certain conditions.

\section{Preliminaries and basic results}

Throughout this article, we let $\mathcal{H}$ be a real Hilbert space which is equipped with an inner product $\langle\cdot, \cdot\rangle$ and the corresponding norm $\|\cdot\|$ and $K$ be a nonempty and closed subset of $\mathcal{H}$. We denote by $d_{K}(\cdot)$ or $d(\cdot, K)$ the usual distance function to the subset $K$, i.e., $d_{K}(u)=$ $\inf _{v \in K}\|u-v\|$. Let us recall the following well-known definitions and some auxiliary results of nonlinear convex analysis and nonsmooth analysis [12-14, 21]. 
Definition 2.1 Let $u \in \mathcal{H}$ be a point not lying in $K$. A point $v \in K$ is called $a$ closest point or a projection of $u$ onto $K$ if $d_{K}(u)=\|u-v\|$. The set of all such closest points is denoted by $P_{K}(u)$, i.e.,

$$
P_{K}(u):=\left\{v \in K: d_{K}(u)=\|u-v\|\right\} .
$$

Definition 2.2 The proximal normal cone of $K$ at a point $u \in K$ is given by

$$
N_{K}^{P}(u):=\left\{\xi \in \mathcal{H}: u \in P_{K}(u+\alpha \xi) \text { for some } \alpha>0\right\} .
$$

Clarke et al. [13], in Proposition 1.1.5, give characterization of $N_{K}^{P}(u)$ as follows.

Lemma 2.1 Let $K$ be a nonempty closed subset in $\mathcal{H}$. Then $\xi \in N_{K}^{P}(u)$ if and only if there exists a constant $\alpha=\alpha(\xi, u)>0$ such that the following proximal normal inequality holds:

$$
\langle\xi, v-u\rangle \leq \alpha\|v-u\|^{2} \quad \text { for all } v \in K .
$$

Definition 2.3 Let $X$ be a real Banach space and $f: X \rightarrow \mathbb{R}$ be Lipschitz with constant $\tau$ near a given point $x \in X$; that is, for some $\varepsilon>0$, we have $|f(y)-f(z)| \leq \tau\|y-z\|$ for all $y, z \in B(x ; \varepsilon)$, where $B(x ; \varepsilon)$ denotes the open ball of radius $\varepsilon>0$ and centered at $x$. The generalized directional derivative of $f$ at $x$ in the direction $v$, denoted as $f^{\circ}(x ; v)$, is defined as follows:

$$
f^{\circ}(x ; v)=\limsup _{y \rightarrow x, t \downarrow 0} \frac{f(y+t v)-f(y)}{t},
$$

where $y$ is a vector in $X$ and $t$ is a positive scalar.

The generalized directional derivative defined earlier can be used to develop a notion of tangency that does not require $K$ to be smooth or convex.

Definition 2.4 The tangent cone $T_{K}(x)$ to $K$ at a point $x$ in $K$ is defined as follows:

$$
T_{K}(x):=\left\{v \in \mathcal{H}: d_{K}^{\circ}(x ; v)=0\right\}
$$

Having defined a tangent cone, the likely candidate for the normal cone is the one obtained from $T_{K}(x)$ by polarity. Accordingly, we define the normal cone of $K$ at $x$ by polarity with $T_{K}(x)$ as follows:

$$
N_{K}(x):=\left\{\xi:\langle\xi, v\rangle \leq 0, \forall v \in T_{K}(x)\right\} .
$$

In 1995, Clarke et al. [14] introduced and studied a new class of nonconvex sets, called proximally smooth sets; subsequently, Poliquin et al. in [21] investigated the aforementioned sets under the name of uniformly prox-regular sets. These have been successfully used in many nonconvex applications in areas such as optimization, economic models, dynamical systems, differential inclusions, etc. For such applications, see $[6-8,10]$. This class seems particularly well suited to overcome the difficulties which arise due to the nonconvexity assumptions on $K$. We take the following characterization proved in [14] as a 
definition of this class. We point out that the original definition was given in terms of the differentiability of the distance function, see [14].

Definition 2.5 For any $r \in(0,+\infty]$, a subset $K_{r}$ of $\mathcal{H}$ is called normalized uniformly proxregular (or uniformly r-prox-regular [21]) if every nonzero proximal normal to $K_{r}$ can be realized by an $r$-ball. This means that for all $\bar{x} \in K_{r}$ and all $0 \neq \xi \in N_{K_{r}}^{P}(\bar{x})$,

$$
\left\langle\frac{\xi}{\|\xi\|}, x-\bar{x}\right\rangle \leq \frac{1}{2 r}\|x-\bar{x}\|^{2}, \quad \forall x \in K_{r} .
$$

Obviously, the class of normalized uniformly prox-regular sets is sufficiently large to include the class of convex sets, $p$-convex sets, $C^{1,1}$ submanifolds (possibly with boundary) of $\mathcal{H}$, the images under a $C^{1,1}$ diffeomorphism of convex sets and many other nonconvex sets, see $[11,14]$.

Lemma 2.2 [14] A closed set $K \subseteq \mathcal{H}$ is convex if and only if it is proximally smooth of radius $r$ for every $r>0$.

If $r=+\infty$, then in view of Definition 2.5 and Lemma 2.2, the uniform $r$-prox-regularity of $K_{r}$ is equivalent to the convexity of $K_{r}$, which makes this class of great importance. For the case of $r=+\infty$, we set $K_{r}=K$.

The following proposition summarizes some important consequences of the uniform prox-regularity needed in the sequel. The proof of this result can be found in [14, 21].

Proposition 2.1 Let $r>0$ and $K_{r}$ be a nonempty closed and uniformly $r$-prox-regular subset of $\mathcal{H}$. Set $U(r)=\left\{u \in \mathcal{H}: d_{K_{r}}(u)<r\right\}$. Then the following statements hold:

(a) For all $x \in U(r), P_{K_{r}}(x) \neq \emptyset$;

(b) For all $r^{\prime} \in(0, r), P_{K_{r}}$ is Lipschitz continuous with constant $\frac{r}{r-r^{\prime}}$ on $U\left(r^{\prime}\right)=\left\{u \in \mathcal{H}: d_{K_{r}}(u)<r^{\prime}\right\}$.

In order to make clear the concept of $r$-prox-regular sets, we state the following concrete example: The union of two disjoint intervals $[a, b]$ and $[c, d]$ is $r$-prox-regular with $r=\frac{c-b}{2}$, see $[9,13,21]$. The finite union of disjoint intervals is also $r$-prox-regular and $r$ depends on the distances between the intervals.

\section{Remarks on nonconvex variational inequalities}

Let $K_{r}$ be a uniformly $r$-prox-regular subset of $\mathcal{H}$. For a given nonlinear single-valued operator $T: \mathcal{H} \rightarrow \mathcal{H}$, Noor [25,26] introduced and considered the problem of finding $u \in K_{r}$ such that

$$
\langle T u, v-u\rangle \geq 0, \quad \forall v \in K_{r} .
$$

Meanwhile, by using Lemma 2.1, he asserted that problem (1) is equivalent to that of finding $u \in K_{r}$ such that

$$
0 \in T u+N_{K_{r}}^{P}(u)
$$

where $N_{K_{r}}^{P}(s)$ denotes the $P$-normal cone to $K_{r}$ at $s$ in the sense of nonconvex analysis. However, this claim is not true in general. 
Remark 3.1 Every solution of problem (1) is a solution of problem (2), but the converse is not necessarily true.

Proof Let $u^{*} \in K_{r}$ be a solution of problem (1). Then we have

$$
\left\langle T u^{*}, v-u^{*}\right\rangle \geq 0, \quad \forall v \in K_{r}
$$

Inequality (3) implies that for all $\alpha>0$,

$$
\left\langle T u^{*}, v-u^{*}\right\rangle+\alpha\left\|v-u^{*}\right\|^{2} \geq 0, \quad \forall v \in K_{r} .
$$

By using (4) and Lemma 2.1, it follows that

$$
-T u^{*} \in N_{K_{r}}^{P}\left(u^{*}\right)
$$

which leads to

$$
0 \in T u^{*}+N_{K_{r}}^{P}\left(u^{*}\right)
$$

The converse of the above statement does not hold in general. Indeed, suppose that inclusion (5) holds for some $u^{*} \in K_{r}$. Then, Lemma 2.1 implies that inequality (4) holds for some $\alpha>0$. However, by using inequality (4), we cannot deduce inequality (3).

The following example illustrates that problem (4) does not imply problem (3).

Example 3.1 Let $\mathcal{H}=\mathbb{R}$ and $K_{r}=[0, \beta] \cup[\gamma, \delta]$ be the union of two disjoint intervals $[0, \beta]$ and $[\gamma, \delta]$, where $0<\beta<\gamma<\delta$. Then $K_{r}$ is an $r$-prox-regular set with $r=\frac{\gamma-\beta}{2}$. Define $T: \mathcal{H} \rightarrow \mathcal{H}$ by

$$
T x=\theta e^{k x} \quad \text { for all } x \in \mathcal{H},
$$

where $k \in \mathbb{R}$ and $\theta<0$ are arbitrary but fixed. Take $u^{*}=\beta$ and let $\alpha \geq-\frac{\theta e^{k \beta}}{\gamma-\beta}$ be arbitrary and fixed. Then, we have

$$
\begin{aligned}
\left\langle T u^{*}, v-u^{*}\right\rangle+\alpha\left\|v-u^{*}\right\|^{2} & =\theta e^{k \beta}(v-\beta)+\alpha(v-\beta)^{2} \\
& =(v-\beta)\left(\alpha(v-\beta)+\theta e^{k \beta}\right), \quad \forall v \in K_{r} .
\end{aligned}
$$

If $v \in[0, \beta]$, then $-\beta \leq \nu-\beta \leq 0$ and

$$
-\alpha \beta+\theta e^{k \beta} \leq \alpha(\nu-\beta)+\theta e^{k \beta} \leq \theta e^{k \beta} .
$$

For $v \in[\gamma, \delta]$, we have $\gamma-\beta \leq \nu-\beta \leq \delta-\beta$ and

$$
\alpha(\gamma-\beta)+\theta e^{k \beta} \leq \alpha(v-\beta)+\theta e^{k \beta} \leq \alpha(\delta-\beta)+\theta e^{k \beta} .
$$

The above facts guarantee that

$$
(v-\beta)\left(\alpha(v-\beta)+\theta e^{k \beta}\right) \geq 0, \quad \forall v \in K_{r} .
$$


Now, (6) and (7) imply that

$$
\left\langle T u^{*}, v-u^{*}\right\rangle+\alpha\left\|v-u^{*}\right\|^{2} \geq 0, \quad \forall v \in K_{r} .
$$

However, it is obvious that $\theta e^{k \beta}(v-\beta)<0$ for all $v \in[\gamma, \delta]$, that is,

$$
\left\langle T u^{*}, v-u^{*}\right\rangle<0 \quad \text { for all } v \in[\gamma, \delta] .
$$

Hence, the inequality

$$
\left\langle T u^{*}, v-u^{*}\right\rangle \geq 0
$$

cannot hold for all $v \in K_{r}$.

In Section 3 of $[25,26]$, the author claimed that problem (1) is equivalent to a fixed point problem.

Lemma 3.1 ([25, 26], Lemma 3.1) $u \in K_{r}$ is a solution of nonconvex variational inequality (1), if and only if $u \in K_{r}$ satisfies the relation

$$
u=P_{K_{r}}[u-\rho T u]
$$

where $\rho>0$ is a constant and $P_{K_{r}}$ is the projection of $\mathcal{H}$ onto the uniformly r-prox-regular set $K_{r}$.

Remark 3.2 By a careful reading, we found that there are two fatal errors in the proof of Lemma 3.1. Firstly, in view of Proposition 2.1, it should be pointed out that for any $r^{\prime} \in(0, r)$, the projection of points in $U\left(r^{\prime}\right)=\left\{u \in \mathcal{H}: d_{K_{r}}(u)<r^{\prime}\right\}$ onto the set $K_{r}$ exists and is unique, that is, for any $x \in U\left(r^{\prime}\right)$, the set $P_{K_{r}}(x)$ is nonempty and singleton. Equation (8) and Proposition 2.1 imply that the point $u-\rho T(u)$ should belong to $U\left(r^{\prime}\right)$ for some $r^{\prime} \in(0, r)$. Unfortunately, it is not necessarily true. Indeed, equation $(8)$ is not necessarily well defined. If $u \in K_{r}$ and $\rho<\frac{r^{\prime}}{1+\|T u\|}$, for some $r^{\prime} \in(0, r)$, then we have

$$
\begin{aligned}
d_{K_{r}}(u-\rho T u) & =\inf _{v \in K_{r}}\|u-\rho T u-v\| \\
& \leq\|u-\rho T u-u\| \\
& =\rho\|T u\| \\
& <\frac{r^{\prime}\|T u\|}{1+\|T u\|}<r^{\prime} .
\end{aligned}
$$

Therefore, $u-\rho T u \in U\left(r^{\prime}\right)$, that is, the set $P_{K_{r}}(u-\rho T u)$ is nonempty and singleton. Hence, in the statement of Lemma 3.1, the constant $\rho$ should be satisfied $\rho<\frac{r^{\prime}}{1+\|T u\|}$ for some $r^{\prime} \in$ $(0, r)$. Secondly, we note that the author $[25,26]$ used the nonconvex variational inclusion (2) as an equivalence formulation of the nonconvex variational inequality (1). However, in view of Remark 3.1 and Example 3.1, the two problems (1) and (2) are not necessarily equivalent. 
Let the operator $T$ be the same as in problem (1). Related to problem (1), Noor [26] considered the problem of finding $z \in \mathcal{H}$ such that

$$
T P_{K_{r}} z+\rho^{-1} Q_{K_{r}} z=0
$$

where $\rho>0$ is a constant, $Q_{K_{r}}=I-P_{K_{r}}$ and $I$ is the identity operator. Problem (9) is called the nonconvex Winer-Hopf equation (NWHE).

Noor [26] claimed that problem (9) is equivalent to problem (1).

Lemma 3.2 ([26], Lemma 4.1) The nonconvex variational inequality (1) has a solution $u \in$ $K_{r}$ if and only if the nonconvex Wiener-Hopf equation (9) has a solution $z \in \mathcal{H}$, satisfying

$$
u=P_{K_{r}} z, \quad z=u-\rho T u,
$$

where $\rho>0$ is a constant.

By a careful reading, we discovered that Lemma 3.1 is the main tool to establish the statement of Lemma 3.2. As it is shown, the statement of Lemma 3.1 is not necessarily true. Consequently, the statement of Lemma 3.2 is not necessarily true.

Since Lemmas 3.1 and 3.2 are the main tools to suggest algorithms and to obtain the results in [25] and [26], in view of the above remarks, the results in $[25,26]$ and the papers where the same technique and method are used, are not valid.

\section{Projection methods and convergence analysis}

Instead of the nonconvex variational inequality (1), in this section, for a given nonlinear operator $T: \mathcal{H} \rightarrow \mathcal{H}$, we consider the problem of finding $u \in K_{r}$ such that

$$
\langle T u, v-u\rangle+\frac{\|T u\|}{2 r}\|v-u\|^{2} \geq 0, \quad \forall v \in K_{r} .
$$

Problem (11) is called the regularized nonconvex variational inequality (RNVI). We prove the equivalence between RNVI (11) and problem (2) as well as fixed point problem (8).

If $r=\infty$, that is, $K_{r}=K$, the convex set in $\mathcal{H}$, then problem (11) collapses to the problem of finding $u \in K$ such that

$$
\langle T u, v-u\rangle \geq 0, \quad \forall v \in K
$$

An inequality of type (12) is called the variational inequality, which was introduced and studied by Stampacchia [1] in 1964.

In the next proposition, the equivalence between nonconvex variational inclusion (2) and regularized nonconvex variational inequality (11) is established.

Proposition 4.1 If $K_{r}$ is a uniformly prox-regular set, then problem (11) is equivalent to problem (2).

Proof Let $u \in K_{r}$ be a solution of problem (11). If $T u=0$, because the vector zero always belongs to any normal cone, we have $0 \in T u+N_{K_{r}}^{P}(u)$. If $T u \neq 0$, then for all $v \in K_{r}$, one has

$$
\langle-T u, v-u\rangle \leq \frac{\|T u\|}{2 r}\|v-u\|^{2} .
$$


Now, Lemma 2.1 implies that $-T u \in N_{K_{r}}^{P}(u)$, and so

$$
0 \in T u+N_{K_{r}}^{P}(u)
$$

Conversely, if $u \in K_{r}$ is a solution of problem (2), then Definition 2.5 guarantees that $u \in K_{r}$ is a solution of problem (11).

Problem (2) is called the nonconvex variational inclusion associated with RNVI (11). Now, by using the projection operator technique, we establish the equivalence between problem (11) and fixed point problem (8).

Lemma 4.1 Let $T$ be the same as in problem (11). Then $u \in K_{r}$ is a solution of problem (11) if and only if $u$ satisfies equation (8), provided that $\rho<\frac{r^{\prime}}{1+\|T u\|}$ for some $r^{\prime} \in(0, r)$.

Proof Let $u \in K_{r}$ be a solution of problem (11). Since $\rho<\frac{r^{\prime}}{1+\|T u\|}$, it follows that equation (8) is well defined. Then, by using Proposition 4.1, we have

$$
\begin{aligned}
0 \in T u+N_{K_{r}}^{P}(u) & \Longleftrightarrow u-\rho T u \in u+\rho N_{K_{r}}^{P}(u) \\
& \Longleftrightarrow u-\rho T u \in\left(I+\rho N_{K_{r}}^{P}\right)(u) \\
& \Longleftrightarrow u=P_{K_{r}}[u-\rho T u],
\end{aligned}
$$

where $I$ is the identity operator and we have used the well-known fact that $P_{K_{r}}=(I+$ $\left.\rho N_{K_{r}}^{P}\right)^{-1}$.

Definition 4.1 An operator $T: \mathcal{H} \rightarrow \mathcal{H}$ is said to be:

(a) monotone if and only if

$$
\langle T u-T v, u-v\rangle \geq 0, \quad \forall u, v \in \mathcal{H} ;
$$

(b) $\kappa$-strongly monotone if and only if there exists a constant $\kappa>0$ such that

$$
\langle T u-T v, u-v\rangle \geq \kappa\|u-v\|^{2}, \quad \forall u, v \in \mathcal{H} ;
$$

(c) $\gamma$-Lipschitz continuous if and only if there exists a constant $\gamma>0$ such that

$$
\|T u-T v\| \leq \gamma\|u-v\|, \quad \forall u, v \in \mathcal{H} .
$$

In the next theorem, the existence and uniqueness of a solution for problem (11) are discussed.

Theorem 4.1 Let the operator $T$ be the same as in problem (11) such that $T$ is $\alpha$-strongly monotone and $\beta$-Lipschitz continuous. If there exists a constant $\rho>0$ satisfying the following conditions:

$$
\rho<\frac{r^{\prime}}{1+\|T u\|} \quad \text { for some } r^{\prime} \in(0, r) \text { and for all } u \in \mathcal{H}
$$


and

$$
\left|\rho-\frac{\alpha}{\beta^{2}}\right|<\frac{\sqrt{\delta^{2} \alpha^{2}-\beta^{2}\left(\delta^{2}-1\right)}}{\delta \beta^{2}}, \quad \delta \alpha>\beta \sqrt{\delta^{2}-1},
$$

where $\delta=\frac{r}{r-r^{\prime}}$, then problem (11) admits a unique solution.

Proof Define $F: K_{r} \rightarrow K_{r}$ by

$$
F(u)=P_{K_{r}}[u-\rho T u], \quad \forall u \in K_{r} .
$$

By using condition (13), we can easily check that the mapping $F$ is well defined. We establish that $F$ is a contraction mapping. For this end, let $u, v \in K_{r}$ be given. From (15) and Proposition 2.1, it follows that

$$
\begin{aligned}
\|F(u)-F(v)\| & =\left\|P_{K_{r}}[u-\rho T u]-P_{K_{r}}[v-\rho T v]\right\| \\
& \leq \delta\|u-v-\rho(T u-T v)\|,
\end{aligned}
$$

where $\delta=\frac{r}{r-r^{\prime}}$. Since the operator $T$ is $\alpha$-strongly monotone and $\beta$-Lipschitz continuous, we get

$$
\begin{aligned}
\|u-v-\rho(T u-T v)\|^{2} & =\|u-v\|^{2}-2 \rho\langle T u-T v, u-v\rangle+\rho^{2}\|T u-T v\|^{2} \\
& \leq\left(1-2 \rho \alpha+\rho^{2} \beta^{2}\right)\|u-v\|^{2}
\end{aligned}
$$

which leads to

$$
\|u-v-\rho(T u-T v)\| \leq \sqrt{1-2 \rho \alpha+\rho^{2} \beta^{2}}\|u-v\| .
$$

Applying (16) and (17), we have

$$
\|F(u)-F(v)\| \leq \theta\|u-v\|
$$

where

$$
\theta=\delta \sqrt{1-2 \rho \alpha+\rho^{2} \beta^{2}}
$$

Condition (14) implies that $\theta<1$. From inequality (18), we infer that $F$ is a contraction mapping. According to the Banach fixed point theorem, there exists a unique point $u^{*} \in$ $K_{r}$ such that $F\left(u^{*}\right)=u^{*}$. It follows from (15) that $u^{*}=P_{K_{r}}\left[u^{*}-\rho T u^{*}\right]$. Now, Lemma 4.1 guarantees that $u^{*} \in K_{r}$ is a solution of problem (11). This completes the proof.

Noor [25] proposed the Mann iteration process for solving problem (1) as follows.

Algorithm 4.1 ([25], Algorithm 3.1) For a given $u_{0} \in \mathcal{H}$, find an approximate solution $u_{n+1}$ by the iterative scheme

$$
u_{n+1}=\left(1-\alpha_{n}\right) u_{n}+\alpha_{n} P_{K_{r}}\left[u_{n}-\rho T u_{n}\right], \quad n=0,1,2, \ldots
$$

where $\alpha_{n} \in[0,1]$ for all $n \geq 0$. 
Noor [26] suggested the following two-step and three-step iterative methods for solving problem (1).

Algorithm 4.2 ([26], Algorithm 3.4) For a given $u_{0} \in K_{r}$, find an approximate solution $u_{n+1}$ by the iterative schemes

$$
\begin{aligned}
& y_{n}=\left(1-\beta_{n}\right) u_{n}+\beta_{n} P_{K_{r}}\left[u_{n}-\rho T u_{n}\right], \\
& u_{n+1}=\left(1-\alpha_{n}\right) u_{n}+\alpha_{n} P_{K_{r}}\left[y_{n}-\rho T y_{n}\right], \quad n=0,1,2, \ldots,
\end{aligned}
$$

where $\alpha_{n}, \beta_{n} \in[0,1]$, for all $n \geq 0$.

Algorithm 4.3 ([26], Algorithm 3.5) For a given $u_{0} \in \mathcal{H}$, find an approximate solution $u_{n+1}$ by the iterative schemes

$$
\begin{aligned}
& y_{n}=\left(1-\gamma_{n}\right) u_{n}+\gamma_{n} P_{K_{r}}\left[u_{n}-\rho T u_{n}\right], \\
& w_{n}=\left(1-\beta_{n}\right) u_{n}+\beta_{n} P_{K_{r}}\left[y_{n}-\rho T y_{n}\right], \\
& u_{n+1}=\left(1-\alpha_{n}\right) u_{n}+\alpha_{n} P_{K_{r}}\left[w_{n}-\rho T w_{n}\right], \quad n=0,1,2, \ldots,
\end{aligned}
$$

where $\alpha_{n}, \beta_{n}, \gamma_{n} \in[0,1]$, for all $n \geq 0$.

Remark 4.1 It should be pointed out that in the context of Algorithms 3.4 and 3.5 from [26], there are minor mistakes. In fact, in iterative processes (3.7) and (3.8) from Algorithm 3.4 in [26], $x_{n}$ must be replaced by $u_{n}$, as we have done in Algorithm 4.2. Meanwhile, in the context of Algorithm 3.5 from [26], $u_{n} \in \mathcal{H}$ must be replaced by $u_{0} \in \mathcal{H}$, as we have done in Algorithm 4.3.

By a careful reading, we found that Algorithms 4.1-4.3 do not work. Indeed, in a way similar to the argument of Remark 3.2, the points $u_{n}-\rho T u_{n}, y_{n}-\rho T y_{n}$ and $w_{n}-\rho T w_{n}$ $(n \geq 0)$ do not belong necessarily to $U\left(r^{\prime}\right)$.

By utilizing Lemma 4.1, we suggest and analyze the following explicit projection iterative methods for solving problem (11).

Algorithm 4.4 Let $T$ be the same as in problem (11) and suppose further that $\rho>0$ is a constant satisfying condition (13). For an arbitrarily chosen initial point $u_{0} \in K_{r}$, compute the iterative sequence $\left\{u_{n}\right\}$ in $K_{r}$ in the following way:

$$
u_{n+1}=P_{K_{r}}\left[u_{n}-\rho T u_{n}\right], \quad n=0,1,2, \ldots
$$

Algorithm 4.5 Let $T$ be the same as in problem (11), and let $\rho>0$ be a constant satisfying condition (13). For an arbitrarily chosen initial point $u_{0} \in K_{r}$, compute the iterative sequence $\left\{u_{n}\right\}$ in $K_{r}$ in the following way:

$$
\begin{aligned}
& y_{n}=P_{K_{r}}\left[u_{n}-\rho T u_{n}\right], \\
& u_{n+1}=P_{K_{r}}\left[y_{n}-\rho T y_{n}\right], \quad n=0,1,2, \ldots
\end{aligned}
$$


Algorithm 4.6 Let $T$ be the same as in problem (11), and let $\rho>0$ be a constant satisfying condition (13). For an arbitrarily chosen initial point $u_{0} \in K_{r}$, compute the iterative sequence $\left\{u_{n}\right\}$ in $K_{r}$ in the following way:

$$
\begin{aligned}
& y_{n}=P_{K_{r}}\left[u_{n}-\rho T u_{n}\right], \\
& w_{n}=P_{K_{r}}\left[y_{n}-\rho T y_{n}\right], \\
& u_{n+1}=P_{K_{r}}\left[w_{n}-\rho T w_{n}\right], \quad n=0,1,2, \ldots
\end{aligned}
$$

We now study the convergence analysis of Algorithm 4.4 and this is the main motivation of our next result.

Theorem 4.2 Let the operator $T$ be the same as in Theorem 4.1, and let all the conditions of Theorem 4.1 hold. Then the iterative sequence $\left\{u_{n}\right\}$ generated by Algorithm 4.4 converges strongly to the unique solution of problem (11).

Proof Theorem 4.1 guarantees the existence of a unique solution $u \in K_{r}$ for problem (11). Hence, Lemma 4.1 implies that $u=P_{K_{r}}[u-\rho T u]$. Then, by using (20) and Proposition 2.1, we have

$$
\begin{aligned}
\left\|u_{n+1}-u\right\| & =\left\|P_{K_{r}}\left[u_{n}-\rho T u_{n}\right]-P_{K_{r}}[u-\rho T u]\right\| \\
& \leq \delta\left\|u_{n}-u-\rho\left(T u_{n}-T u\right)\right\| \\
& \leq \theta\left\|u_{n}-u\right\| \leq \theta^{2}\left\|u_{n-1}-u\right\| \leq \cdots \leq \theta^{n+1}\left\|u_{0}-u\right\|,
\end{aligned}
$$

where $\theta$ is the same as in (19). Since $\theta<1$, it follows that the right-hand side of the above inequality tends to zero as $n \rightarrow \infty$, whence we deduce that $u_{n} \rightarrow u$ as $n \rightarrow \infty$. This completes the proof.

In a similar way to the proof of Theorem 4.2, one can prove the strong convergence of the iterative sequence $\left\{u_{n}\right\}$ generated by Algorithms 4.5 and 4.6.

\section{Wiener-Hopf equations technique}

In this section, by utilizing Lemma 4.1 and the projection method, the equivalence between NWHE (9) and RNVI (11) is established. By using the obtained equivalence formulation, some iterative algorithms for solving RNVI (11) are suggested and analyzed. The convergence analysis of the proposed iterative algorithms under some certain conditions is studied.

Let the operator $T$ be the same as in problem (11). Related to problem (11), we consider the problem of finding $z \in \mathcal{H}$ satisfying (9).

If $r=\infty$, then problem (9) is equivalent to finding $z \in \mathcal{H}$ such that

$$
T P_{K} z+\rho^{-1} Q_{K} z=0
$$

where $Q_{K}=I-P_{K}$. Equation (22) is the original Wiener-Hopf equation mainly due to Shi [22]. 
Remark 5.1 It was shown that the Wiener-Hopf equations had played an important and significant role in developing several numerical techniques for solving variational inequalities and related optimizations problems (see, for example, $[18,19,22]$ and the references therein).

In the next lemma, the equivalence between RNVI (11) and NWHE (9) is proved.

Lemma 5.1 Let $T$ be the same as in problem (11). Then $u \in K_{r}$ is a solution of RNVI (11) if and only if NWHE (9) has a solution $z \in \mathcal{H}$ satisfying (10), provided that $\rho<\frac{r^{\prime}}{1+\|T u\|}$ for some $r^{\prime} \in(0, r)$.

Proof Let $u \in K_{r}$ be a solution of problem (11). Since $\rho<\frac{r^{\prime}}{1+\|T u\|}$ for some $r^{\prime} \in(0, r)$, it follows from Lemma 4.1 that

$$
u=P_{K_{r}}[u-\rho T u] .
$$

Taking $z=u-\rho T u$ in (23), we have

$$
u=P_{K_{r}} z
$$

By using (24) and the fact that $z=u-\rho T u$, we have

$$
z=P_{K_{r}} z-\rho T P_{K_{r}} z
$$

Obviously, the above equation is equivalent to

$$
T P_{K_{r}} z+\rho^{-1} Q_{K_{r}} z=0
$$

where $Q_{K_{r}}=I-P_{K_{r}}$, that is, $z \in \mathcal{H}$ is a solution of NWHE (9).

Conversely, if $z \in \mathcal{H}$ is a solution of NWHE (9), satisfying

$$
u=P_{K_{r}} z, \quad z=u-\rho T u,
$$

then Lemma 4.1 implies that $u \in K_{r}$ is a solution of RNVI (11). This completes the proof.

Noor [26] used the equivalence formulation between the two problems (1) and (9) and suggested the following iterative methods for solving problem (1).

Algorithm 5.1 ([26], Algorithm 4.1) For a given $z_{0} \in \mathcal{H}$, compute $z_{n+1}$ by the iterative schemes

$$
\begin{aligned}
& u_{n}=P_{K_{r}} z_{n}, \\
& z_{n+1}=\left(1-\alpha_{n}\right) z_{n}+\alpha_{n}\left(u_{n}-\rho T u_{n}\right), \quad n=0,1,2, \ldots,
\end{aligned}
$$

where $0 \leq \alpha_{n} \leq 1$, for all $n \geq 0$ and $\sum_{n=0}^{\infty} \alpha_{n}=\infty$. 
Algorithm 5.2 ([26], Algorithm 4.2) For a given $z_{0} \in \mathcal{H}$, compute $z_{n+1}$ by the iterative schemes

$$
\begin{aligned}
& u_{n}=P_{K_{r}} z_{n}, \\
& z_{n+1}=\left(1-\alpha_{n}\right) z_{n}+\alpha_{n}\left(u_{n}-T u_{n}+\left(1-\rho^{-1}\right) Q_{K_{r}} z_{n}\right), \quad n=0,1,2, \ldots,
\end{aligned}
$$

where $0 \leq \alpha_{n} \leq 1$, for all $n \geq 0$ and $\sum_{n=0}^{\infty} \alpha_{n}=\infty$.

Algorithm 5.3 ([26], Algorithm 4.3) For a given $z_{0} \in \mathcal{H}$, compute $z_{n+1}$ by the iterative scheme

$$
z_{n+1}=\left(1-\alpha_{n}\right) z_{n}+\alpha_{n}\left(I-\rho^{-1} T^{-1}\right) Q_{K_{r}} z_{n}, \quad n=0,1,2, \ldots,
$$

where $0 \leq \alpha_{n} \leq 1$, for all $n \geq 0$ and $\sum_{n=0}^{\infty} \alpha_{n}=\infty$.

Remark 5.2 As it is pointed out, the two problems (1) and (9) are not necessarily equivalent. Hence, the equivalence between problems (1) and (9) cannot be used for suggesting Algorithms 5.1-5.3 to approximate the solution of problem (1). Even without considering the mentioned fact, we note that Algorithms 5.1-5.3 do not work. Indeed, in a way similar to the argument of Remark 3.2, iterative scheme (25) is well defined provided that for each $n \geq 0$, the point $z_{n}$ belongs to $U\left(r^{\prime}\right)$ for some $r^{\prime} \in(0, r)$. Accordingly, $z_{0}$ must be taken in $U\left(r^{\prime}\right)$ for some $r^{\prime} \in(0, r)$. However, for a given $z_{0} \in U\left(r^{\prime}\right)$, iterative scheme (26) does not guarantee that $z_{n} \in U\left(r^{\prime}\right)$ for each $n>0$, because $U\left(r^{\prime}\right)$ is not necessarily convex.

The following example illustrates that for any given uniformly $r$-prox-regular set $K_{r}$ in $\mathcal{H}$ and $r^{\prime} \in(0, r)$, the set $U\left(r^{\prime}\right)$ in $\mathcal{H}$ is not necessarily convex.

Example 5.1 Let $\mathcal{H}$ and $K_{r}$ be the same as in Example 3.1. As has been mentioned in Example 3.1, $K_{r}$ is an $r$-prox-regular set with $r=\frac{\gamma-\beta}{2}$. Let $r^{\prime} \in(0, r)$ be arbitrary but fixed. Then we have

$$
U\left(r^{\prime}\right)=\left\{u \in \mathcal{H}: d_{K_{r}}(u)<r^{\prime}\right\}=\left(-r^{\prime}, \beta+r^{\prime}\right) \cup\left(\gamma-r^{\prime}, \delta+r^{\prime}\right),
$$

which is clearly nonconvex.

By using NWHE (9) and Lemma 5.1, we get a fixed point formulation to construct a new projection iterative algorithm for solving RNVI (11).

By using (9) and (10), we have

$$
\begin{aligned}
T P_{K_{r}} z+\rho^{-1} Q_{K_{r}} z=0 & \Longleftrightarrow Q_{K_{r}} z=-\rho T P_{K_{r}} z \\
& \Longleftrightarrow z=P_{K_{r}} z-\rho T P_{K_{r}} z \\
& \Longleftrightarrow z=u-\rho T u .
\end{aligned}
$$

This fixed point formulation enables us to construct the following iterative algorithm for solving RNVI (11). 
Algorithm 5.4 Let the operator $T$ be the same as in RNVI (11) and assume further that $\rho>0$ is a constant satisfying condition (13) for some $r^{\prime} \in(0, r)$. For a given $z_{0} \in U\left(r^{\prime}\right)$, compute the iterative sequence $\left\{z_{n}\right\}$ in $U\left(r^{\prime}\right)$ in the following way:

$$
\left\{\begin{array}{l}
u_{n}=P_{K_{r}} z_{n}, \\
z_{n+1}=u_{n}-\rho T u_{n}, \quad n=0,1,2, \ldots
\end{array}\right.
$$

We now apply Lemma 5.1 and study the convergence analysis of Algorithm 5.4.

Theorem 5.1 Let the operator $T$ be the same as in Theorem 4.1 and suppose that all the conditions of Theorem 4.1 hold. Assume further that $\rho>0$ is a constant satisfying conditions (13) and (14). Then there exists $z \in \mathcal{H}$ such that $z$ is a solution of problem (9) and the sequence $\left\{z_{n}\right\}$ generated by Algorithm 5.4 converges strongly to $z$.

Proof Theorem 4.1 guarantees that RNVI (11) admits a unique solution $u \in K_{r}$. Hence Lemma 5.1 implies the existence of a unique point $z \in U\left(r^{\prime}\right)$ satisfying (10). By using (10), (27) and the assumptions, we have

$$
\begin{aligned}
\left\|z_{n+1}-z\right\| & \leq\left\|u_{n}-u-\rho\left(T u_{n}-T u\right)\right\| \\
& \leq \sqrt{1-2 \rho \alpha+\rho^{2} \beta^{2}}\left\|u_{n}-u\right\| .
\end{aligned}
$$

From (10) and (27) and Proposition 2.1, it follows that

$$
\left\|u_{n}-u\right\|=\left\|P_{K_{r}} z_{n}-P_{K_{r}} z\right\| \leq \delta\left\|z_{n}-z\right\|,
$$

where $\delta$ is the same as in (14). Substituting (29) in (28), we have

$$
\left\|z_{n+1}-z\right\| \leq \theta\left\|z_{n}-z\right\|
$$

where $\theta$ is the same as in (19). Applying (30), we deduce that

$$
\left\|z_{n+1}-z\right\| \leq \theta\left\|z_{n}-z\right\| \leq \theta^{2}\left\|z_{n-1}-z\right\| \leq \cdots \leq \theta^{n+1}\left\|z_{0}-z\right\| .
$$

Condition (14) implies that $\theta<1$. Since $\theta \in(0,1)$, it follows that the right-hand side of the above inequality tends to zero as $n \rightarrow \infty$, which implies that the sequence $\left\{z_{n}\right\}$ generated by Algorithm 5.4 converges strongly to $z$. This completes the proof.

\section{Concluding remarks}

In this paper, we have investigated and analyzed the nonconvex variational inequality (2.1) from $[25,26]$ and the Wiener-Hopf equation (2.5) from [26]. We have proved that the problem $(2.1)$ from $[25,26]$ is not equivalent to the fixed point problem $(3.1)$ from $[25,26]$ and the Wiener-Hopf equation (2.5) from [26]. That is, Lemma 3.1 in $[25,26]$ is incorrect. Lemma 3.1 in $[25,26]$ is the main key to suggest the Mann iteration processes in $[25,26]$ and to establish the strong convergence of the iterative sequences generated by the proposed algorithms in $[25,26]$. Since Lemma 3.1 in $[25,26]$ is not valid, the algorithms and results in $[25,26]$ are also not valid. Indeed, we have pointed out that unlike the claim of 
the author in $[25,26]$, the existence and uniqueness of a solution for nonconvex variational inequality $(1)((2.1)$ in $[25,26])$ up to now have not been established and still remain an open problem. We have also pointed out that the Mann iteration processes proposed by the author in $[25,26]$ for solving problem (1) do not work. To overcome these problems in $[25,26]$, we have introduced and considered the regularized nonconvex variational inequality (RNVI) (11) and the nonconvex Wiener-Hopf equation (NWHE) (9). By using the projection operator technique, we have verified the equivalence between RNVI (11) and the fixed point problem (8) as well as NWHE (9). By using the obtained equivalences, we have proved the existence of a unique solution for RNVI (11) and suggested and analyzed some explicit projection iterative methods for solving RNVI (11). The convergence analysis of the proposed iterative schemes under some suitable conditions has also been studied. But the two following questions have not been replied and still remain open problems:

(1) Can the existence of a solution for nonconvex variational inequality (1) be proved?

(2) Can the Mann iteration process for solving nonconvex variational inequality (11) be presented?

Competing interests

The authors declare that they have no competing interests.

Authors' contributions

All authors contributed equally and significantly in this paper. All authors read and approved the final manuscript.

\section{Author details}

1Department of Mathematics, Sari Branch, Islamic Azad University, Sari, Iran. ${ }^{2}$ Department of Mathematics Education, Kyungnam University, Changwon, Gyeongnam 631-701, Korea.

\section{Acknowledgements}

This work was supported by the Basic Science Research Program through the National Research Foundation (NRF) Grant funded by the Ministry of Education of the Republic of Korea (2013R1A1A2054617). The authors thank the anonymous referees for their constructive comments which contributed to the improvement of the present paper.

Received: 16 June 2013 Accepted: 27 September 2013 Published: 11 Nov 2013

References

1. Stampacchia, G: Formes bilineaires coercitives sur les ensembles convexes. C. R. Acad. Sci. Paris 258, 4413-4416 (1964)

2. Bensoussan, A, Lions, JL: Application des Inéquations Variationelles en Control et en Stochastiques. Dunod, Paris (1978)

3. Glowinski, R, Letallec, P: Augmented Lagrangian and Operator-Splitting Methods in Nonlinear Mechanics. SIAM, Philadelphia (1989)

4. Harker, PT, Pang, JS: Finite-dimensional variational inequality and nonlinear complementarity problems: a survey of theory, algorithm and applications. Math. Program. 48, 161-220 (1990)

5. Baiocchi, C, Capelo, A: Variational and Quasi Variational Inequalities. Wiley, New York (1984)

6. Bounkhel, M: Existence results of nonconvex differential inclusions. Port. Math. (N.S.) 59, 283-309 (2002)

7. Bounkhel, M: General existence results for second order nonconvex sweeping process with unbounded perturbations. Port. Math. 60, 269-304 (2003)

8. Bounkhel, M, Azzam, L: Existence results on the second order nonconvex sweeping processes with perturbations. Set-Valued Anal. 12, 291-318 (2004)

9. Bounkhel, M, Tadji, L, Hamdi, A: Iterative schemes to solve nonconvex variational problems. J. Inequal. Pure Appl. Math. 4, 1-14 (2003)

10. Bounkhel, $M$, Thibault, L: Nonconvex sweeping process and prox-regularity in Hilbert space. J. Nonlinear Convex Anal. 6, 359-374 (2005)

11. Canino, A: On p-convex sets and geodesics. J. Differ. Equ. 75, 118-157 (1988)

12. Clarke, FH: Optimization and Nonsmooth Analysis. Wiley, New York (1983)

13. Clarke, FH, Ledyaev, YS, Stern, RJ, Wolenski, PR: Nonsmooth Analysis and Control Theory. Springer, New York (1998)

14. Clarke, FH, Stern, RJ, Wolenski, PR: Proximal smoothness and the lower $C^{2}$ property. J. Convex Anal. 2, 117-144 (1995)

15. Giannessi, F, Maugeri, A: Variational Inequalities and Network Equilibrium Problems. Plenum, New York (1995)

16. Giannessi, F, Maugeri, A, Pardalos, PM: Equilibrium Problems, Nonsmooth Optimization and Variational Inequalities Problems. Kluwer Academic, Dordrecht (2001)

17. Gilbert, RP, Panagiotopoulos, PD, Pardalos, PM: From Convexity to Nonconvexity. Kluwer, Dordrecht (2001)

18. Lions, PL, Mercier, B: Splitting algorithms for the sum of two nonlinear operators. SIAM J. Numer. Anal. 16, 964-979 (1979)

19. Lions, JL, Stampacchia, G: Variational inequalities. Commun. Pure Appl. Math. 20, 493-512 (1967) 
20. Moudafi, A: Projection methods for a system of nonconvex variational inequalities. Nonlinear Anal., Theory Methods Appl. 71, 517-520 (2009)

21. Poliquin, RA, Rockafellar, RT, Thibault, L: Local differentiability of distance functions. Trans. Am. Math. Soc. 352, 5231-5249 (2000)

22. Shi, P: Equivalence of variational inequalities with Wiener-Hopf equations. Proc. Am. Math. Soc. 111, 339-346 (1991)

23. Bernard, F, Thibault, L, Zlateva, N: Characterizations of prox-regular sets in uniformly convex Banach spaces. J. Convex Anal. 13, 525-559 (2006)

24. Bernard, F, Thibault, L, Zlateva, N: Prox-regular sets and epigraphs in uniformly convex Banach spaces: various regularities and other properties. Trans. Am. Math. Soc. 363, 2211-2247 (2011)

25. Noor, MA: Projection methods for nonconvex variational inequalities. Optim. Lett. 3, 411-418 (2009)

26. Noor, MA: Some iterative methods for nonconvex variational inequalities. Comput. Math. Model. 21, 97-108 (2010)

10.1186/1029-242X-2013-531

Cite this article as: Balooee and Kim: Some remarks on regularized nonconvex variational inequalities. Journal of Inequalities and Applications 2013, 2013:531

\section{Submit your manuscript to a SpringerOpen ${ }^{\odot}$ journal and benefit from:}

- Convenient online submission

- Rigorous peer review

- Immediate publication on acceptance

- Open access: articles freely available online

- High visibility within the field

- Retaining the copyright to your article 